\title{
Online Collaborative Design within a Web-enabled Environment
}

\author{
Daizhong Su, Jiansheng Li and Shuyan Ji \\ Advanced Design and Manufacturing Engineering Centre, SBE \\ The Nottingham Trent University, UK \\ E-mail: \{daizhong.su, jiansheng.li, shuyan.ji2\}@ntu.ac.uk \\ URL: http://www.admec.ntu.ac.uk
}

\begin{abstract}
A Web-enabled environment (WEE) for online collaboration has been which combines appropriate communications and interconnectivity tools to allow designers to interactively communicate over the Internet in real time, regardless of their IT platforms. The detailed structure of the WEE is presented, followed by description of the key techniques for online collaborative design within the WEE: remote execution of large size executable programs, data file exchange between different CAD systems, and data sharing in real time with distant users.
\end{abstract}

\section{Introduction}

As the Internet becomes more reliable and widespread, it offers the possibility to enable rapid collaborative product design and manufacture between partners almost regardless of their geographical locations. Much research has been undertaken to achieve this aim using Web-based distributed collaborative environments and related technologies with some, albeit limited, success. Name and Eaglestein listed the tools for a distributed environment [1], Roy et al reported the development of a prototype Web-based collaborative product modeling system [2], Adapalli and Addepalli described different ways of integrating manufacturing process simulations by mean of the Web [3], Kim et al developed a system to store STEP data in an object-oriented database and covert STEP data into VRML data [4], Huang et al studied the Web techniques that can be used for developing collaborative systems [5], Chen et al investigated into a network-supported collaborative design over the Internet/Intranet based on dynamic data exchange [6,7], and Lee et al presented a Web-enabled approach for feature-based modeling in a distributed design environment [8]. The authors' research team has been actively involved in this area, for example, Su et al conducted research in network support for integrated design [9,10,11], and developed a multi-user Internet environment for gear design optimization [12,13,14]; Hull et al developed a software tool for collaborative design and manufacture over the Internet [15].

The processes of design, analysis and manufacture are increasingly requiring very specialised expertise, and may be performed on sites which are distant from each other. The communication of the wealth of relevant engineering information required be- 
tween such sites is limited at present in that the data transfer speed can be slow and the hardware and software platforms at each site must be compatible. In order to overcome the limitations, this paper presents a structure for a Web-enabled collaborative environment which combines appropriate communications and interconnectivity tools to allow designers to interactively communicate in real time, regardless of their IT platforms.

Nowadays, product design data is not only managed by the design and production activities [16] but also used in the later stages of the product life cycle [17], which is important to obtain the agility in manufacturing required to improve the competitiveness of companies [18]. The technical data associated with products are different from business data since complex semantics are required, which thus make it very difficult to exchange between different design systems [19].

To meet the challenges mentioned above, a Web-enabled environment and associated techniques have been developed by the authors for geographically dispersed designers to collaborate online. In the following sections, the Web-enabled environment is presented first, followed by two associated techniques: remote execution of large size programs/packages and Web-enable collaborative CAD.

\section{The Web-enabled Environment}

The Web-enabled environment (WEE) addresses the issue of Internet connections and communications. Its main objective is for the teams/enterprises geographically remotely located to collaborate in design and manufacture with the aid of the Internet and related techniques. Therefore, it is essential to establish an effective means for communication over the Internet. To achieve this, the WEE for collaboration is developed.

\subsection{Features of the WEE}

To develop the WEE, it has to be considered that the partners are not only dispersed geographically but may also work with different platforms, operating systems, protocols and languages. The currently existing ICT tools/systems in this field can address some aspects, such as accessing remote databases, invocation to the remote processes or sharing and integrating of multiple data resources, which are not enough for an integrated heterogeneous environment. As a large heterogeneous platform for collaboration and integration over the Internet, the WEE has the following features.

1. Scalability -- The system architecture can accommodate any growth in future load such as new computer processors and/or architectures and tools.

2. Openness -- The system can be easily extended and modified. Any new components integrated in the system can communicate and work together with some of components that already exist in the system. 
3. Heterogeneity -- The system is constructed using different programming languages, operated on different hardware platforms and obeys different protocols. Heterogeneous components have to communicate with each other and be interoperated.

4. Resources access and inter-operation -- Resources including software and data should be accessible to, and operated by, all partners.

5. Legacy codes reusability -- Existing applications can be integrated seamlessly together with a new application without code-rewriting and can be interoperated with each other. The reusable components enable efficient development processes and reliable application systems.

6. Artificial intelligence -- Artificial neural networks, fuzzy logic and genetic algorithms are utilized for the control of process and conflict checking.

The combination of all the above to provide a robust tool for Web-based collaborative environment for design and manufacture is a novel contribution of the research.

\subsection{Three Tier Architecture of the WEE}

The Web-enabled environment presented in this paper is constructed based on the CORBA (common object request broker architecture) technology, because CORBA provides an excellent communication mechanism between client and server. The environment consists of three tiers: A User tier, a Web server tier and an Application tier as shown in Figure 1.

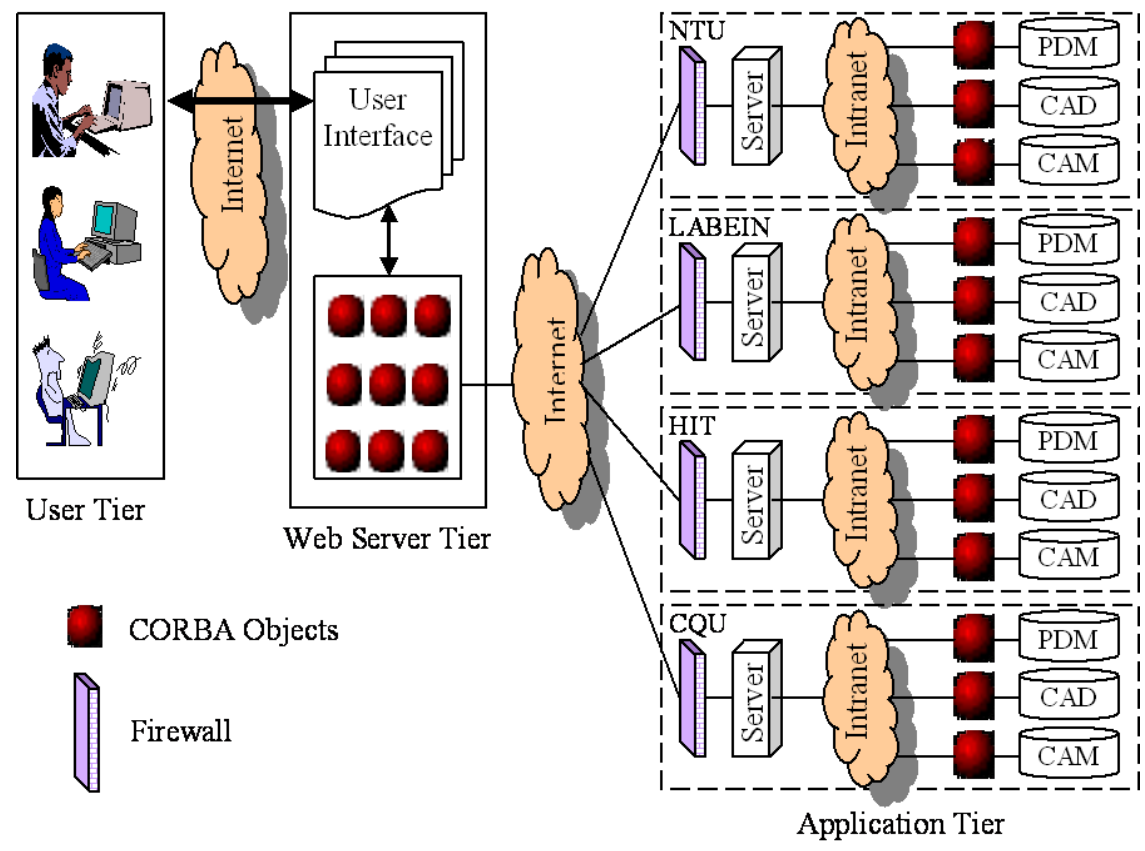

Fig. 1. Architecture of WEE 
Each tier has the following functions:

1. User tier-Valid customers of the WEE are allowed to visit HTML or XML pages located on the Web server tier over the Internet. The user needs to know only how to use the graphic interface and does not to have to be aware of the technical details behind this.

2. Web server tier-This consists of graphical user interface, CORBA agent object and intelligent control of virtual design systems.

- The user interface includes the whole activities flow according to the design and manufacturing process and Web service tools. All these Web pages contain the inside linkage to the CORBA agent objects.

- CORBA agent objects are in charge of linking and finding the essential CORBA agent objects associated with the remote applications or software and activating them and retrieving the results. Through CORBA all the components, including applications, software or objects dispersed on the remote heterogeneous systems, can be encapsulated into the objects that CORBA agent objects can manage and find. Therefore the components amongst different partners can be found, shared and interoperated by each other.

- The artificial intelligent control is being accomplished by employing artificial neural networks, fuzzy logic and genetic algorithms for the intelligent control of the whole process of design and manufacture. It includes the CAD/CAM connection and feature recognition, intelligent distribution with neural networks, intelligent scheduling using GA and virtual and evaluative activities.

- In addition there are synchronous and non-synchronous communication.

3. Application tier-This tier consists of a number of applications and tools needed to implement the design and manufacturing, and services that will be provided by CORBA objects. These applications may be dispersed geographically and also written in different languages, work on different platforms and operating systems, and are encapsulated into components that the CORBA agent can find. The interface of each application, mapped into a CORBA agent object, is separated from the implementation written in respective languages/packages that a partner prefers to use. All these applications consist of different large computing programs, various kinds of PDM, CAD and CAM software, accessing and searching applications for backend database resources such as SQL, DBMS or JDBC, and other resources from customer services.

\subsection{Development of the WEE}

In order to implement the integration and collaboration amongst the partners, the environment has to be provided with the following modules.

- Integration of application

- Communication and interoperation between partners

- Unifying graphical user interface

- Intelligent process management 
The user interface is developed mainly in Java combined with other appropriate Web technologies. Applications necessary to the design and manufacture are implemented by all the partners using their own systems, which will ensure the use of heterogeneous systems.

Integration of applications without rewriting legacy codes is one of the key issues on which the environment being developed is based. CORBA is an open, vendorneutral, middleware standard that allows the proliferating number of hardware and software applications to communicate with one another using heterogeneous systems. CORBA is a complete distributed object computing framework to extend applications across networks, languages, component boundaries and operating systems. Distributed collaborative systems which are constructed based on CORBA/Java have features such as high-performance, scalability, maturity, inter-operability, support for legacy systems and ease of development. This research utilizes the object technology CORBA, Java and other technologies in the development of the Web-enabled environment.

\section{Effective Remote-execution of Large Size Programs/Packages}

In order to achieve best product design and low production costs, some large-sized programs, such as design optimisation and finite element analysis software, are often used in the design phase of product development. Usually, such programs/packages are time-consuming in computation and may not be valid to download due to software copyright, or due to their large size and the limited network bandwidth. To remotely execute such software in an effective way in order to conduct on-line collaborative design within the WEE, an approach has been developed which is presented below.

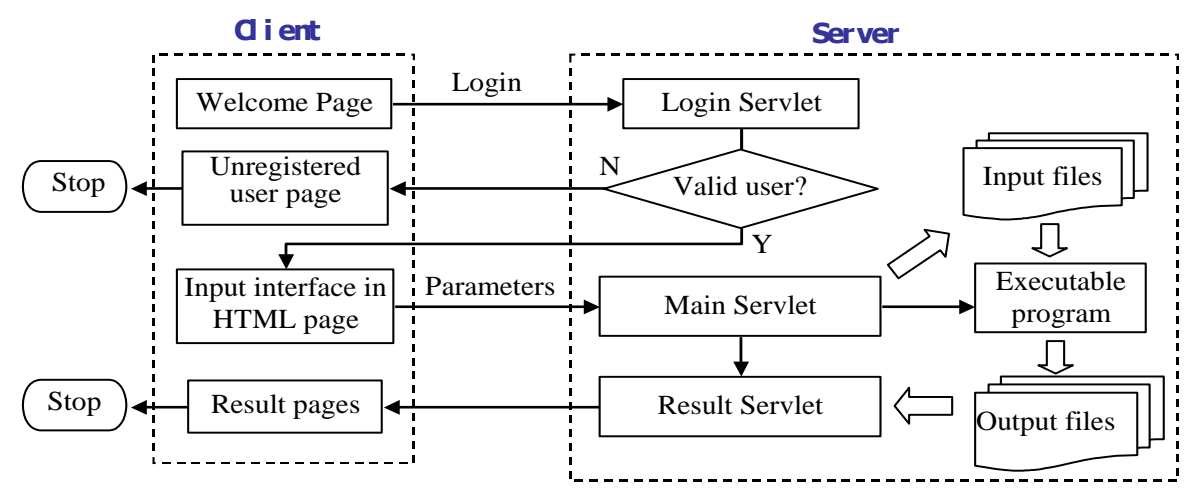

Fig. 2. Remote execution environment based on Java Servlets

\subsection{Application of Java Servlets Technology in the System}

Java Servlets technology is applied to remotely execute a large sized program over the Internet and to improve the execution speed within the WEE. The executable program 
is located and executed on the server-side and the results are sent to the client-side after the completion of the program. To accomplish this, a combination of Java Servlets, HTML, JavaScript, Java, HTTP protocol and multi-user environment are utilized.

The structure of the system is shown in Figure 2. The user on the client side inputs the parameters of the program via the input interface in the HTML page. Then these parameters are sent to the server, which calls the Servlets located on the server. When the user clicks on the submit button on the HTML page, a Servlets program is activated by the HTML code. It parses the parameters and writes them into the input files and invokes the executable program which is located on the server. When the execution is completed and output files are created, Servlets return the results to the client.

\subsection{Application of CORBA Technology in the System}

CORBA technology is used to provide a better solution for this remote execution environment. The CORBA's ORB (Object Request Broker) is utilized as the communication bus for all objects in the system. It enables objects to transparently make requests to---and receive responses from---objects located locally or remotely. The client is not aware of the mechanisms used to communicate with, activate, or store the server objects.

The IDL (Interface Definition Language) is used to define interfaces in CORBA. An IDL interface file describes the data types and methods or operations that a server provides for implementation of a given object. IDL is not a programming language; it describes interfaces only, but has no relation to implementation. The IDL can be mapped to various programming languages, including $\mathrm{C}, \mathrm{C}++$, Smalltalk Ada, COBOL and Java.

Figure 3 portrays the architecture of the remote execution environment where CORBA is used as Middleware for the interface between the client GUI written using Java Applet and executable programs such as $\mathrm{C} / \mathrm{C}++$ applications distributed on the Web. ORB helps to turn a local application (distributed) into the Web-based object, so that it can be accessed over the network such as the Intranet, Entranet or Internet.

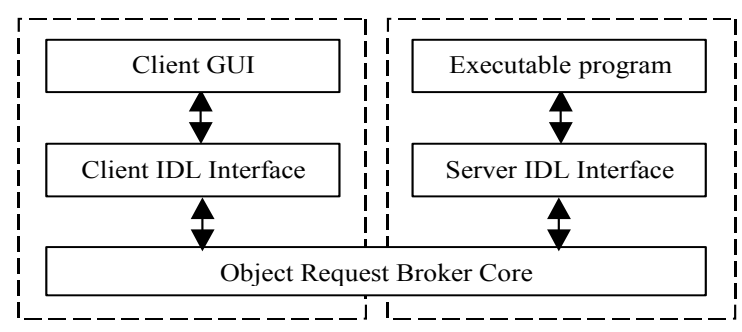

Fig. 3. Architecture of WEE

The client GUI (graphical user interface) captures any user input, and then it uses the CORBA software component, called the ORB, to transparently invoke and pass the parameters to the executable program at the application server. The results of execution are then passed from the server back to the client GUI. 


\section{Web-based Collaborative Computer Aided Design}

In an advanced manufacturing model, all sorts of efficient processes have a close relationship with the geometric model of product, which is the foundation of the operation of an integrated design and manufacturing system. Many commercial CAD and finite element analysis (FEA) software packages, such as Pro-E, UG, SolidWorks, Auto$\mathrm{CAD}$, ANSYS, etc, are widely used in product design and manufacturing. Although all of these programs support 3-dimensional modeling, the data format is different in each of them. In the distributed collaborative environment considered by this research, a given partner may have different CAD and FEA software from those other partners may have. In order to collaborate with each other, it is necessary to implement robust and reliable methodologies for the exchange and sharing of data between heterogeneous CAD systems.

To achieve this, a neutral file approach for CAD data transfer and a CORBA based approach for CAD data sharing over the Internet are proposed as detailed below.

\subsection{Neutral File Approach for CAD Data Transfer}

Neutral files and neutral file interfaces are needed in order to exchange product data between CAD systems. Direct translators exist but the number of required translators becomes too large if there are many CAD systems involved in the data transfer. For each pair of CAD systems to be able to communicate, two translators are required, one for each direction. For a new additional CAD system, several translators have to be added to each existing CAD system. Figure 4(a) shows the situation using direct translators. When using a neutral file format only one pre- and post-processor is needed for each CAD system. When a new CAD system is added, only one pre- and postprocessor needs to be added. Figure 4(b) shows the situation for data transfer using a neutral file format.

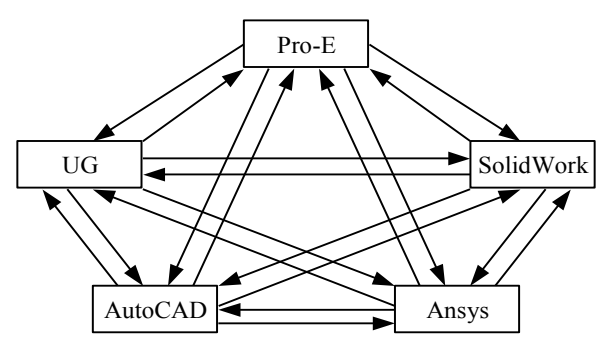

(a)

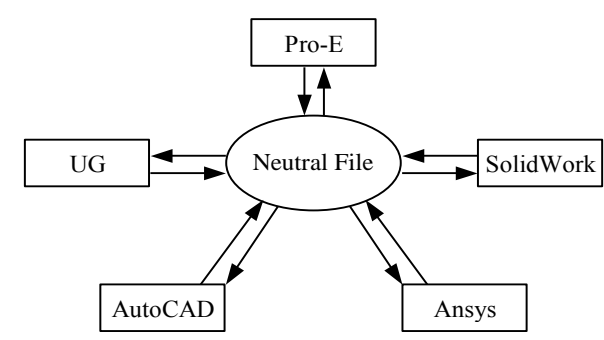

(b)

Fig. 4. CAD data transfer: (a) direct data transfer (b) using a neutral file 


\subsection{Web-based CAD Data Sharing}

In order to conduct integrated design over the Internet/Intranet within the Webenabled environment, it is necessary to provide an online framework to enable geographically dispersed team members to discuss and to modify CAD data simultaneously.

Using currently available commercial Internet communication software, such as Windows Netmeeting, two or more users can share their CAD drawings by remote desktop sharing. However, massive bitmap data needs to be transferred across the Internet, so that the speed of interaction is greatly constrained by the limited network bandwidth. Moreover, such software can only run on the same operating system. To solve this problem, a Web-based approach for collaborative computer aided design across different operating systems is developed within the WEE. Figure 5 shows the working flow chart for this system.

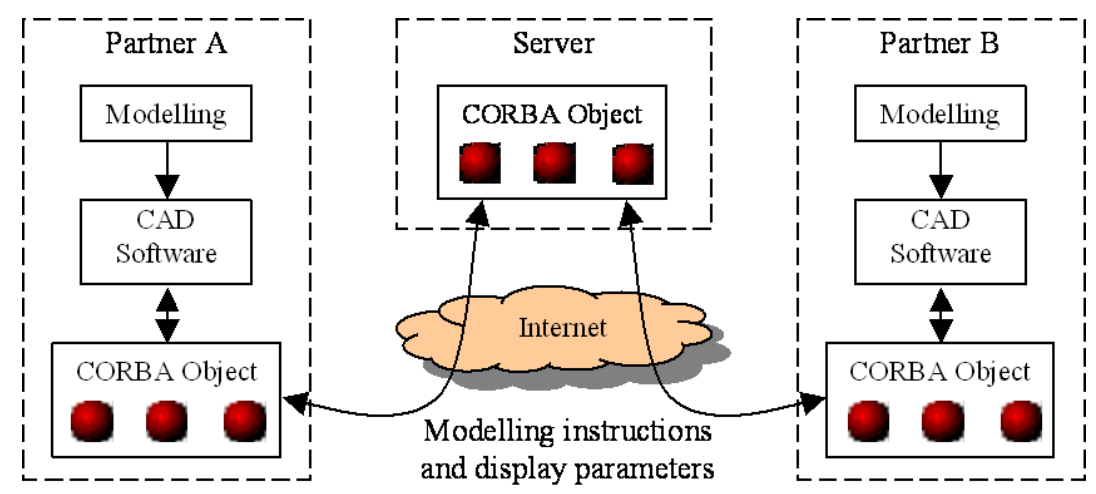

Fig. 5. Flow chart of CAD sharing system

To reduce the load on network transportation, this research puts forward a new approach for real time CAD data sharing. In this new approach, users in different locations start their own process instance with the same CAD software, and connect to the same server by Internet communications that follow CORBA rules. The information transferred through the Internet is not the bitmap data, but the design instructions and display parameters so that the quantity of the Internet transferred data can be greatly reduced and, therefore, the response speed of this proposed collaborative design environment increases manifold.

Assuming partner $\mathrm{A}$ is sharing $\mathrm{CAD}$ data and collaborating with partner $\mathrm{B}$, when partner A develops a model using a particular CAD software, the modelling instructions and display parameters will be transferred to the server in the meantime, and then, from that server to partner B's computer and displayed using the same CAD software. When user B works on the model, the process is reversed. In this way, partners $\mathrm{A}$ and $\mathrm{B}$ can share their $\mathrm{CAD}$ data in real time. 


\section{Concluding Remarks}

A Web-enabled collaborative environment has been presented in this paper. The three tier structure enables the environment's advanced features such as scalability, openness, heterogeneity, resources accessibility, legacy codes reusability and artificial intelligence. The key techniques involved in the development of the software environment include CORBA broker for collaboration and remote design using Java Servlets.

The approach developed for remote-execution of large size programs/packages provides an effective tool for remotely accessing software without downloading which is beneficial for utilizing the resources amongst geographically dispersed collaborative teams. In the approach, a combination of Java Servlets, HTML, JavaScript, Java, HTTP protocol and multi-user environment are utilized.

Different from existing systems/methods, the Web-based collaborative CAD presented in this paper utilises neutral files and neutral file interfaces to exchange product data between CAD systems, which has the advantages that only one pre- and postprocessor is needed for each CAD system. To reduce the load on network transportation, a new approach for real time CAD data sharing is presented. In the approach, only the design instructions and display parameters are transferred over the Internet, so that the quantity of the Internet transferred data can be greatly reduced and the response speed increases.

\section{Acknowledgement}

This research is supported by the EU Asia IT\&C programme (Grant No. ASI/B7301/3152-099/71553) and Asia-Link programme (Grant No. ASI/B7-301/98/679023), which has been carried out at The Nottingham Trent University, UK in cooperation with Harbin Institute of Technology and Chongqing University in China, Foundation LABEIN in Spain and Lappeenranta University of Technology in Finland.

\section{References}

1. Name, E.V., Eagelstein, G.: The Wired Engineer: Emerging Technologies and the Designer. ANTEC'98 (1998) 3052 - 3055

2. Roy, U., Bharadwaj, B., Kodkani, S., Cargian, M.: Product Development in a Collaborative Design Environment. Concurr Engng: Res Appl, Vol.5, No.4 (1997) 347 - 365

3. Adapalli, S., Addepalli, K.: World Wide Web Integration of Manufacturing Process Simulations. Concurr: Practice Experience, Vol.9, No.11 (1997) 1341-1350

4. Kim, C.Y., Kim, N., Kim, Y., Kang, S.H., O'Grady, P.: Distributed Concurrent Engineering: Internet-Based Interactive 3-D Dynamic Browsing and Markup of STEP Data. Concurr Engng: Res Appl, Vol.6, No.1 (1998) 53 - 70

5. Huang, G.Q., Lee, S.W., Mak, K.L.: Web-Based Product and Process Data Modeling in Concurrent Design for X. Robotics Comput-Integrated Manufact, Vol.15 (1999) 53-63 
6. Chen, X., Su, D., Li, Z.: Network-Supported Collaborative Design Based On Dynamic Data Exchange. Proceedings of the International Conference on Computer Aided Industrial Design and Conceptual Design, 16-20 October 2001, Jinan, China (2001) 448-452

7. Chen, X., Yin, Y., Su, D.: Collaborative Computer Aided Design over the Internet/Intranet. Proceedings of the International Conference on e-Commerce Engineering: New Challenges for Global Manufacturing in 21st Century, 16-18 September 2001, Xi'an, China (2001) RID06-1

8. Lee, J.Y., Kim, H., Han, S.B.: Web-Enabled Feature-Based Modeling in a Distributed Design Environment. CD-ROM Proceedings of the 1999 ASME Design Engineering Technical Conferences and Computers and Information in Engineering Conference, Las Vegas, Nevada (1999)

9. Su, D., Chen, X.: Network Support for Integrated Design. Integrated Manufacturing Systems - The International Journal of Manufacturing Technology Management, Vol.14, No.6 (2003) 537-546

10. Su, D., Ji, S., Li, J., Hull, J.B.: Web-enabled Collaborative Environment for Integrated Design and Manufacture. Proceedings of International Conference on Concurrent Engineering, 27-31 July 2002, Cranfield, UK (2002) 93-101

11. Su, D., Amin, N., Chen, X., Wang, Y.: Internet/Intranet Based Integrated Design of Mechanical Transmission Systems. 8th Drive and Control Conferences, 13-15 March 2001, London, UK (2001) 13-20

12. Su, D., Ji, S., Amin, N., Hull, J.B.: An Internet-Based System of Gear Design Optimization Using Java Servlets. Proceedings of the International Conference on Computer Aided Industrial Design and Conceptual Design, 16-20 October 2001, Jinan, China (2001) 30-36

13. Su, D., Ji, S., Amin, N., Hull, J.B.: Multi-User Internet Environment for Gear Design Optimisation. Integrated Manufacturing Systems - The International Journal of Manufacturing Technology Management, Vol.14, No.6 (2003) 498-507

14. Amin, N., Su, D.: Enhancement of Speed and Efficiency of an Internet Based Gear Design Optimisation. International Journal of Automotive Technology and Management, Vol.3, No.3/4 (2003) 279-292

15. Hull, J. B., Su, D., Ji, S.: Development of a Powerful Software Tool for Collaborative Design and Manufacture over the Internet. Proceedings of the International Conference on Industrial Tools, 8-12 April 2003, Bled, Slovenia (2003) 399-402

16. Yoo, S.B., Kim, Y.: Web-Based Knowledge Management for Sharing Product Data in Virtual Enterprises. International Journal of Production Economics, Vol.7, No.2 (2002) 173183

17. Hardwick, M., Spooner, D., Rando, T., Morris, K.C.: Sharing Manufacturing Information in Virtual Enterprises. Communications of the ACM, Vol.39, No.2 (1996) 46-54

18. Gunasekaran, A.: Agile Manufacturing: A Framework for Research and Development. International Journal of Production Economics, Vol.62, No.1/2 (1999) 87-105

19. McKay, A., Bloor, M., Pennington, A.: A Framework for Product Data. IEEE Transactions on Knowledge and Data Engineering, Vol.8, No.5 (1996) 825-838 